Hydrol. Earth Syst. Sci., 12, 1189-1200, 2008

www.hydrol-earth-syst-sci.net/12/1189/2008/

(C) Author(s) 2008. This work is distributed under

the Creative Commons Attribution 3.0 License.

\title{
A stochastic approach for the description of the water balance dynamics in a river basin
}

\author{
S. Manfreda and M. Fiorentino \\ Dipartimento di Ingegneria e Fisica dell'Ambiente (DIFA), Università degli Studi della Basilicata, \\ via dell'Ateneo Lucano, 10, Potenza, 85100, Italy
}

Received: 17 January 2008 - Published in Hydrol. Earth Syst. Sci. Discuss.: 13 March 2008

Revised: 9 July 2008 - Accepted: 18 August 2008 - Published: 17 September 2008

\begin{abstract}
The present paper introduces an analytical approach for the description of the soil water balance dynamics over a schematic river basin. The model is based on a stochastic differential equation where the rainfall forcing is interpreted as an additive noise in the soil water balance. This equation can be solved assuming known the spatial distribution of the soil moisture over the basin transforming the twodimensional problem in space in a one dimensional one. This assumption is particularly true in the case of humid and semihumid environments, where spatial redistribution becomes dominant producing a well defined soil moisture pattern. The model allowed to derive the probability density function of the saturated portion of a basin and of its relative saturation. This theory is based on the assumption that the soil water storage capacity varies across the basin following a parabolic distribution and the basin has homogeneous soil texture and vegetation cover. The methodology outlined the role played by the soil water storage capacity distribution of the basin on soil water balance. In particular, the resulting probability density functions of the relative basin saturation were found to be strongly controlled by the maximum water storage capacity of the basin, while the probability density functions of the relative saturated portion of the basin are strongly influenced by the spatial heterogeneity of the soil water storage capacity. Moreover, the saturated areas reach their maximum variability when the mean rainfall rate is almost equal to the soil water loss coefficient given by the sum of the maximum rate of evapotranspiration and leakage loss in the soil water balance. The model was tested using the results of a continuous numerical simulation performed with a semi-distributed
\end{abstract}

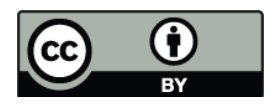

Correspondence to: S. Manfreda (manfreda@unibas.it) model in order to validate the proposed theoretical distributions.

\section{Introduction}

Dynamics of soil moisture in time and space is governed by complex and dynamical interactions between climate, soil and vegetation. Its spatial distribution over a river basin provides a crucial link between hydrological and ecological processes through its controlling influence on runoff generation, groundwater recharge, transpiration, carbon assimilation, etc. The interrelationship between ecological and geophysical determinants of surface water balance is at the forefront of a number of outstanding issues in ecohydrological science (e.g. Rodríguez-Iturbe and Porporato, 2004; Montaldo et al., 2005).

Recent research has received significant inputs for the description of this variable through the numerous experimental campaigns carried out in the last years (e.g. Monsoon, 1990; Washita, 1992, 1994; SGP, 1997, 1999; Tarrawarra experiment). These experiments have increased our understanding of the temporal variability and of the spatial structure of the soil moisture fields and of the importance of physical characteristics such as soil texture, vegetation and topographic patterns for soil moisture variability (Western et al., 2002; Kim and Barros, 2002; Wilson et al., 2004; Jawson and Niemann, 2007).

The dynamics of soil moisture at a point has been extensively investigated by numerous authors using stochastic differential equations to derive its steady-state probability density function (e.g. Rodríguez-Iturbe et al., 1999; Laio et al., 2001; Porporato et al., 2004; Rigby and Porporato, 2006).

Published by Copernicus Publications on behalf of the European Geosciences Union. 
These theories have been useful to describe the vegetation water stress in a probabilistic framework (Porporato et al., 2001) and to investigate on the interactive manner by which resource availability are manifested within various ecological systems observed in nature (e.g. van Wijk and Rodríguez-Iturbe, 2002; Scanlon et al., 2005; Caylor et al., 2005).

Recent studies have extended the theoretical description of the soil moisture to the spatial scale introducing a spacetime soil moisture model driven by a stochastic space-time rainfall forcing described by a sequence of circular cell of Poisson rate (Isham et al., 2005; Rodríguez-Iturbe et al., 2006; Manfreda et al., 2006). The methodology explicitly accounts for soil characteristics, vegetation patterns, and rainfall dynamics neglecting topographical effects and the upper bound due to soil saturation. This soil moisture model can be considered representative of a relatively flat landscape under arid/semiarid climatic conditions.

The description of the soil moisture evolution over a river basin is, at the moment, a challenging topic that may be useful for both ecohydrological and hydrological research. Some examples in this direction are given in the paper by Botter et al. (2007a), where the probability density functions of the slow components of the runoff are derived using a river basin schematization with uniform macroscopic parameters governing the soil water balance neglecting the spatial heterogeneity of soil properties. The same authors extended the previous work introducing the spatial heterogeneity of the basin summing the runoff contributions provided by different subbasins with spatially averaged soil properties (Botter et al., 2007b), but still each subbasin is considered as an homogeneous entity where the relative saturation follows the same dynamics of a point process.

The present work represents an attempt to fill such a gap introducing a mathematical schematization for the derivation of the probability distribution of the relative saturation of a basin accounting for the spatial heterogeneity in soil water storage capacity. The proposed scheme includes a number of approximations, but it leads to an interesting framework for the derivation of the main statistics of basin scale variables. Among others, our interest focused on the behaviour of relative saturation and saturated areas over a river basin that may be responsible of the dynamics of riparian vegetation as well as runoff generation.

The theory is based on the conceptual Xinanjiang model that describes watershed heterogeneity using a parabolic curve for the distribution of the soil water storage capacity (Zhao et al., 1980). The Xinanjiang model was first developed in 1973 and published in English in 1980. It is a well-known lumped watershed model widely used in China. Furthermore, the adopted relationship between the extent of saturated areas and the volume stored in the catchment has driven the evolution of a number of more recent models such as the Probability Distributed Model (Moore and Clarke, 1981; Moore, 1985, 1999), the
VIC model (Wood et al., 1992) and the ARNO model (Todini, 1996).

The present paper provides a description of the mathematical framework used to derive the probability density functions of the soil water content and of the portion of saturated areas at basin scale in Sects. 2 and 3. Results of the theory along with an application of the model are discussed in Sect. 4 that precedes the conclusions.

\section{Model description}

\subsection{Rainfall model}

Rainfall occurrences are modelled by a sequence of instantaneous pulses that occur in a Poisson process of rate $\lambda$ in time and uniform in space. Each pulse is characterized by a random total depth $h$ exponentially distributed with mean $\alpha$ that may be considered as the mean daily rainfall since the model is interpreted at the daily time scale (see Rodríguez-Iturbe et al., 1999).

In the following, we will refer to a normalized version of the density function of rainfall depths described as

$f_{H}(h)=\gamma e^{-\gamma h}$

where $\gamma=w_{\max } / \alpha$ and $w_{\max }$ is the maximum value of the soil water storage capacity in the basin.

The spatial heterogeneity of rainfall is neglected assuming uniform distribution of rainfall occurring at random in time over the entire basin. Such an assumption may be more or less reliable depending on climatic characteristics of rainfall forcing and basin size. In general, one should expect that it becomes more realistic for medium/small size basins. The climatic conditions may also affect the spatial correlation and the extend of rainfall fields that become more and more uniform in humid regions.

2.2 The variability of the soil water storage capacity over the basin

The water storage capacity of the soil is certainly one of the most significant parameter for a correct description of soil moisture dynamics. In fact, it is the main factor controlling the temporal dynamics of the process as clearly shown by Manfreda and Rodríguez-Iturbe (2006). For this reason, in the present work, the soil thickness is assumed to vary over the basin according to a given distribution. For sake of simplicity, the remaining sources of heterogeneity like pattern of vegetation and soil texture variability will be neglected in the present work assuming that the soil texture as well as the vegetation are uniform over the watershed. 
The watershed heterogeneity is described using a parabolic curve for the water storage capacity of the soil (Zhao et al., 1980)

$$
\frac{f}{F}=1-\left(1-\frac{W}{w_{\max }}\right)^{b}
$$

where $f / F$ represents the fraction of the basin with water storage capacity $\leq W, w_{\max }$ is the maximum value of the water storage capacity in the basin and $b$ is a shape parameter that according to Zhao (1992) assumes values between 0.10.4 increasing with the characteristic dimension of the basin. An example of the Eq. (2) is given in Fig. 1 where this function is plotted for different values of $b$. The parameter $b$ affects the spatial heterogeneity of $W$ that increases with larger values of $b$ and becomes a uniform distribution when $b=0$.

The above distribution has been extensively used in several conceptual models where the parameters $b$ and $w_{\max }$ have been calibrated against runoff data.

A first attempt to seek for a physical interpretation of the parameters $b$ and $w_{\max }$ was made by Sivapalan and Woods (1995). In this work, the authors observed a qualitative connection between the landform types and the soil depths of a basin in Western Australia to the topographic position. Later on, Sivapalan et al. (1997) developed a conceptual rainfall/runoff model along the lines of the VIC model (Wood et al., 1992) exploiting the topographic index of the TOPMODEL $\left(W_{I}=\ln (a / \tan \beta)\right.$ by Beven and Kirkby, 1979) to define the parameters of Eq. (2).

In a more recent work, Chen et al. (2007) stated that Eq. (2) can be estimated directly from digital elevation data. In particular, they use the spatial distribution of the TOPMODEL topographic index to estimate the so called index of runoff generation difficulty $\left(\mathrm{IRDG}=\left(\max \left[W_{I}\right]-W_{I}\right) /\left(\max \left[W_{I}\right]-\min \left[W_{I}\right]\right)\right)$ through a normalized function of the topographic index as suggested by Gou et al. (2000). Specifically, the authors propose to substitute the parabolic curve of soil water storage capacity of the Xinanjiang model with the cumulative frequency distribution of IRDG. Under this hypothesis one can estimate the shape parameter $b$ by fitting Eq. (2) with the cumulative frequency distribution of IRDG.

In the following, we provide a sequence of definitions useful for model description and comprehension.

The total water storage capacity of the basin is obtained integrating $(1-f / F)$ between $W=0$ and $w_{\max }$, obtaining

$\mathrm{WM}=\frac{w_{\max }}{1+b}$,

that according to Zhao (1984) and Zhao and Wang (1988) assumes values between $120 \mathrm{~mm}$ and $160 \mathrm{~mm}$ depending on the climatic zone.

In order to obtain a water balance equation with only one state variable, it is necessary to make the hypothesis that the soil water distribution is known over the basin. In particular, it is possible to assume that the soil water content is

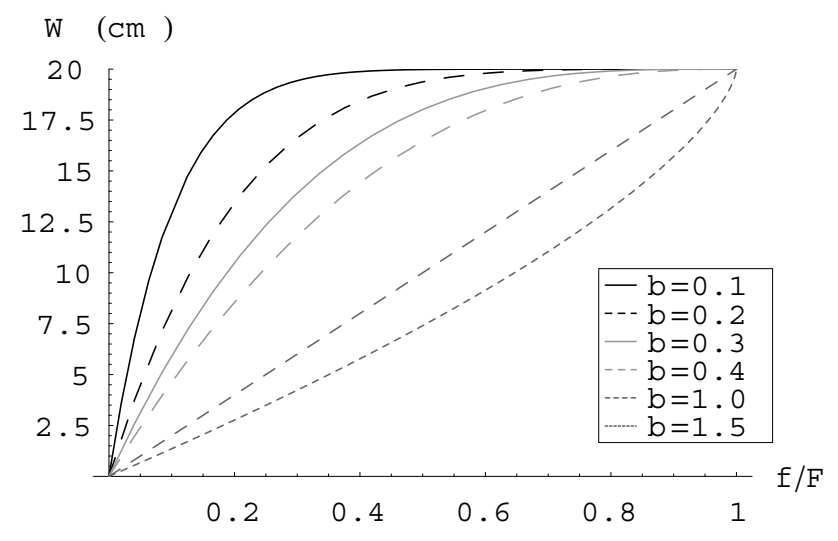

Fig. 1. Distribution of the water storage capacity of a river basin assuming $w_{\max }=20 \mathrm{~cm}$, while the parameter $b$ changes from 0.1 to 1.5 .

redistributed within the basin cumulating in the areas with lower soil depth following the same assumption of the $\mathrm{Xi}$ nanjiang model. The conceptual schematization of the basin is sketched in Fig. 2, where both the soil water content distribution and the soil water storage capacity are described. From this graph, it is also clear that the relative saturated areas, $a$, are described by the same relationship given in Eq. (2) where $a$ correspond to the ratio $f / F$.

The watershed-average soil moisture storage at time $t$, is the integral of $1-f / F$ between zero and the actual value of the water level in the basin scheme, $w m_{t}$,

$W_{t}=\int_{0}^{w m_{t}}\left(1-\frac{f}{F}\right) d W=W M\left(1-\left(1-\frac{w m_{t}}{w_{\max }}\right)^{1+b}\right)$.

The relative saturation of the basin is a significant variable to interpret the basin dynamics and it will be considered, from now on, the state variable of the system along with the saturated portion of the basin, $a$. The relative saturation of the basin may be defined as the ratio between the total water content of the basin divided by the total water storage capacity. Under the described schematization, the relative saturation of the basin, $s$, can be defined as

$s=\frac{W_{t}}{W M}=\left(1-\left(1-\frac{w m_{t}}{w_{\max }}\right)^{1+b}\right)$.

\subsection{The soil water losses}

The function describing the soil water losses represents the deterministic part of the stochastic equation describing the soil water balance. It depends on the local value of the soil water content and the maximum rate of soil water losses. The main contributions to soil losses are given by: the actual evapotranspiration and the soil leakage. A possible approximation for the sum of this two terms is given by a linear 

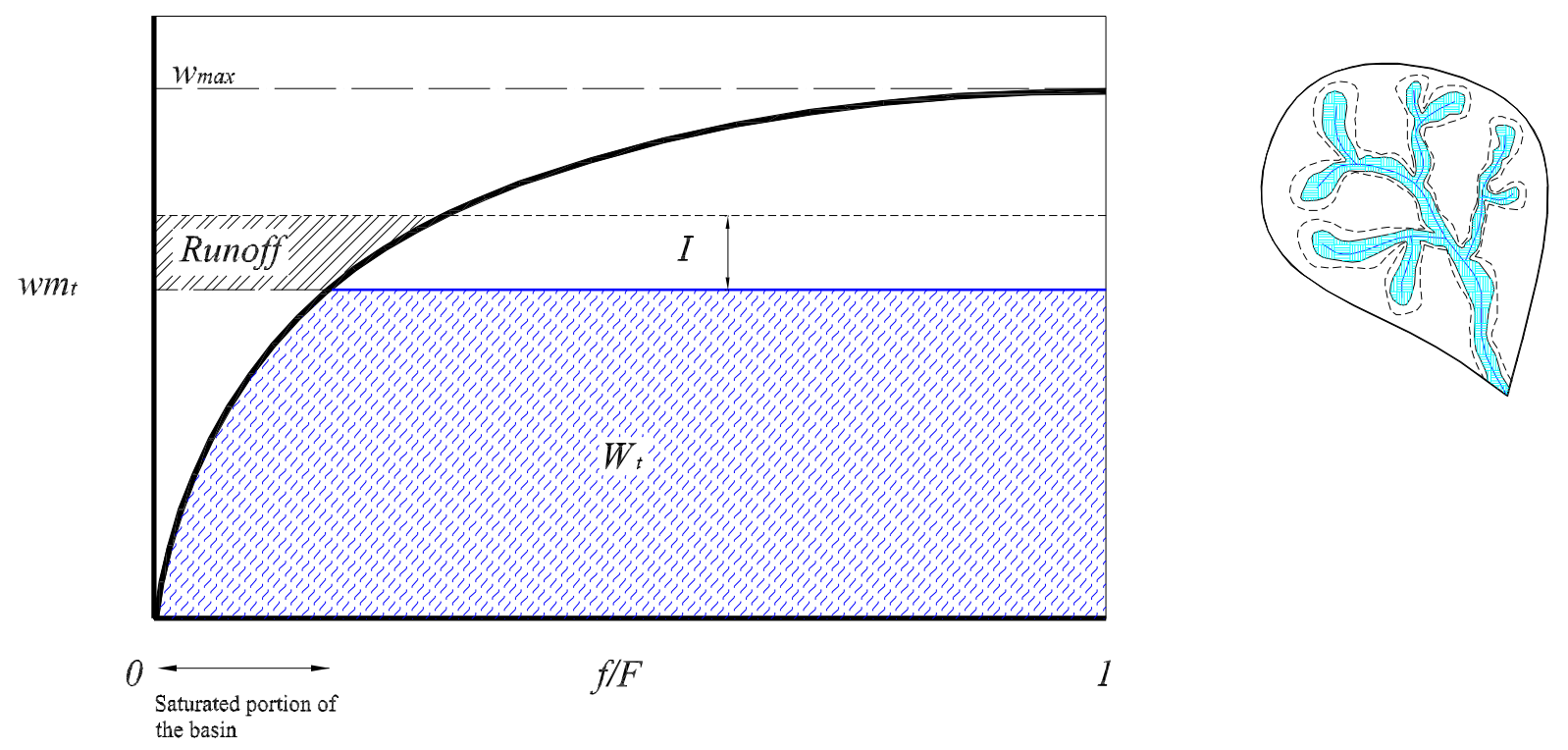

Fig. 2. Schematization of the basin structure and soil water content distribution. The black line represents the distribution of the soil water storage capacity, $W$, that ranges from 0 to $w_{\max }$; the blue line depicts the water distribution over the schematic basin whose level is $w m_{t}$; the dashed line depicts the increase in $w m_{t}$ after a rainfall event producing an infiltration $I$ over the unsaturated portion of the basin, while the saturated and the becoming saturated portion of the basin will produce a runoff represented by the dashed area of the graph.

function where the soil losses are assumed to be proportional to the relative saturation of soil in a point

$L(\zeta)=V \zeta(t, x)$,

where $L(\zeta)$ is the soil water loss relative to the relative soil saturation $\zeta(t, x)$ at time $t$ in the point $x$ in space, and $V$ is the water loss coefficient.

It is worth nothing to remark that the same loss function has been used by numerous authors (e.g. Entekhabi and Rodríguez-Iturbe, 1994; Pan et al., 2003; Porporato et al., 2004; Rodríguez-Iturbe et al., 2006) essentially for two reasons: first of all, it represents a reasonable approximation for the sum of the actual evapotranspiration and the leakage and second it is a useful simplification in a mathematical framework.

Since the adopted soil loss function is linear, it may be generalized at the basin scale using the product between the relative basin saturation, $s$, and the water loss coefficient. It follows

$L_{b}\left(w m_{t}\right)=V s=V\left(1-\left(1-\frac{w m_{t}}{w_{\max }}\right)^{1+b}\right)$.

For analytical purposes, the soil water losses can be expressed as a function of the relative water level in the basin expressed through the ratio $R=\frac{w m_{t}}{w_{\max }}$. In this case, the soil water losses are computed, using an approximated exponential function, as

$L_{b}\left(R=\frac{w m_{t}}{w_{\max }}\right) \cong V\left(\frac{e^{-k R}-1}{e^{-k}-1}\right)$ where $k$ is a coefficient that has been used to fit the above with Eq. (7). The two functions were fitted imposing the condition that they subtend the same area between $R=0$ and 1. Using this assumption, one may obtain the following expression

$b=\frac{2-2 e^{k}+k+e^{k} k}{e^{k}-k-1}$,

that may be solved numerically in $k$ providing an estimate of $k$ as a function of the parameter $b$. This yields $k \cong$ $b /\left(\frac{b}{7}-\frac{1}{3}\right)$.

The Eq. (9) is represented in Fig. 3 for different values of the parameter $b$ that varies from 0.1 up to an hypothetical value of 1.5. This graph shows how the soil water loss function at the basin scale becomes more non-linear with the increase of the values of $b$.

\section{The water balance equation}

The water balance equation needs to be written at the basin scale in order to derive the relative dynamics of soil water. This problem can tackled working with the mass conservation equation of total water, $W_{t}$, or with the water level in the parabolic reservoir. In the first case, the rainfall forcing represents a multiplicative noise, while in the second case it is an additive noise. This last approach is consequently preferable for analytical purposes. 
Using the above approximations for the rainfall forcing and for the spatial distribution of the soil water storage capacity, the soil water balance over the basin can be described through the following stochastic differential equation in $w m_{t}$

$$
\frac{d w m_{t}}{d t}=I-V s
$$

where $I$ represents an additive term of infiltration and water losses are assumed to be proportional to the relative saturation of the basin $s$. The advantage to solve the water balance equation in $w m_{t}$ is that the infiltration rate can be summed as an additive term of the stochastic differential equation. The water level $w m_{t}$ in the basin schematization increases as long as the infiltration does not exceed the maximum water storage capacity of the basin $w_{\max }$, but this does not mean that there no runoff production. The infiltration is equal to the rainfall depth in the portion of the basin that have a residual water storage capacity available to be filled, there after the rainfall will be converted into runoff (see Fig. 2). The schematization, in fact, accounts for the upper bound imposed by the soil saturation.

In the present scheme, the runoff generation occur for saturation excess in the saturated portion of the basin obtaining a behaviour comparable with a Dunne mechanism where the direct precipitation on saturated areas (saturated overland flow) is dominant runoff generation mechanisms (e.g. Hibbert, 1967; Dunne and Black, 1970).

The water balance equation can be solved using the standardized variable

$R=\frac{w m_{t}}{w_{\max }}$,

where $R \in[0,1]$ that represents the relative water level in the parabolic reservoir describing the basin.

Under these assumptions, it is convenient to standardize the soil water loss rate

$\rho(R)=\beta\left(\frac{e^{-k R}-1}{e^{-k}-1}\right)$,

where $\beta=V /\left(w_{\max }\right)$ is the normalized soil water loss coefficient.

The water balance equation becomes

$\frac{d R}{d t}=\frac{I}{w_{\max }}-\rho(R)$.

Following Rodríguez-Iturbe et al. (1999), the probability density function (PDF) of $R$ can be obtained and solved analytically for steady-state conditions. The PDF of $R$, obtained using the simplified loss function $\rho(R)$ in the water balance equation above, becomes

$$
\begin{aligned}
& p(R)=\frac{C}{\rho(R)} e^{-\gamma R+\lambda \int \frac{1}{\rho(R)} d u}= \\
& \frac{C e^{k(R-1)-R \gamma}\left(e^{k}-1\right)\left(e^{k R}-1\right)^{\frac{\lambda\left(1-e^{-k}\right)}{k \beta}}-1}{\beta},
\end{aligned}
$$

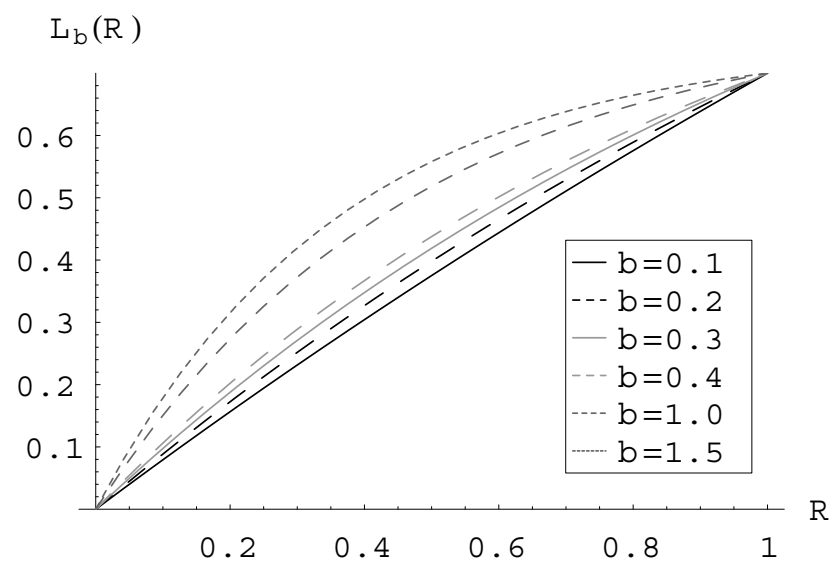

Fig. 3. Soil water losses computed at the basin scale as a function of the ratio $R=\frac{w m_{t}}{w_{\max }}$.

where $C$ is a constant of integration that may be computed simply imposing the normalizing condition, $\int_{0}^{1} p(R) d R=1$. Thus, $C$ assumes the following value

$$
C=1 / \int_{0}^{1} \frac{e^{k(-1+R)-R \gamma}\left(e^{k}-1\right)\left(e^{k R}-1\right)^{-1+\frac{\lambda-e^{-k \lambda}}{k \beta}}}{\beta} d R
$$

$$
\begin{aligned}
& C=\frac{\beta}{\frac{\Gamma\left[1-\frac{\gamma}{k}\right] \Gamma\left[\frac{\lambda-e^{-k \lambda}}{k \beta}\right]}{k e^{k} \Gamma\left[\frac{k \beta-\beta \gamma+\lambda-e^{-k} \lambda}{k \beta}\right]}+\frac{F_{1}\left[1-\frac{\gamma}{k}, 1+\frac{\left(e^{-k}-1\right) \lambda}{k \beta}, 2-\frac{\gamma}{k}, e^{k}\right]}{e^{\gamma}(\gamma-k)}} . \\
& \frac{1}{(-1)^{\frac{\lambda-e^{-k}}{k \beta}}\left(e^{k}-1\right)}
\end{aligned}
$$

where $\Gamma[$.$] is the complete Gamma Function and F_{1}[., ., .,$. is the Hypergeometric Function (Abramowitz and Stegun, 1964; Prudnikov et al., 1986).

According to Eq. (2), the water level in the parabolic reservoir proposed to describe the soil water storage capacity can be related to the fraction of saturated areas, $a$, as

$w m_{t}=\left(1-(1-a)^{\frac{1}{b}}\right) w_{\max }$,

or to the relative saturation of the basin using Eq. (6). Consequently, the derivation of the probability density function at the steady-state of $a$ and $s$ can be obtained straightforward as derived distributions. 


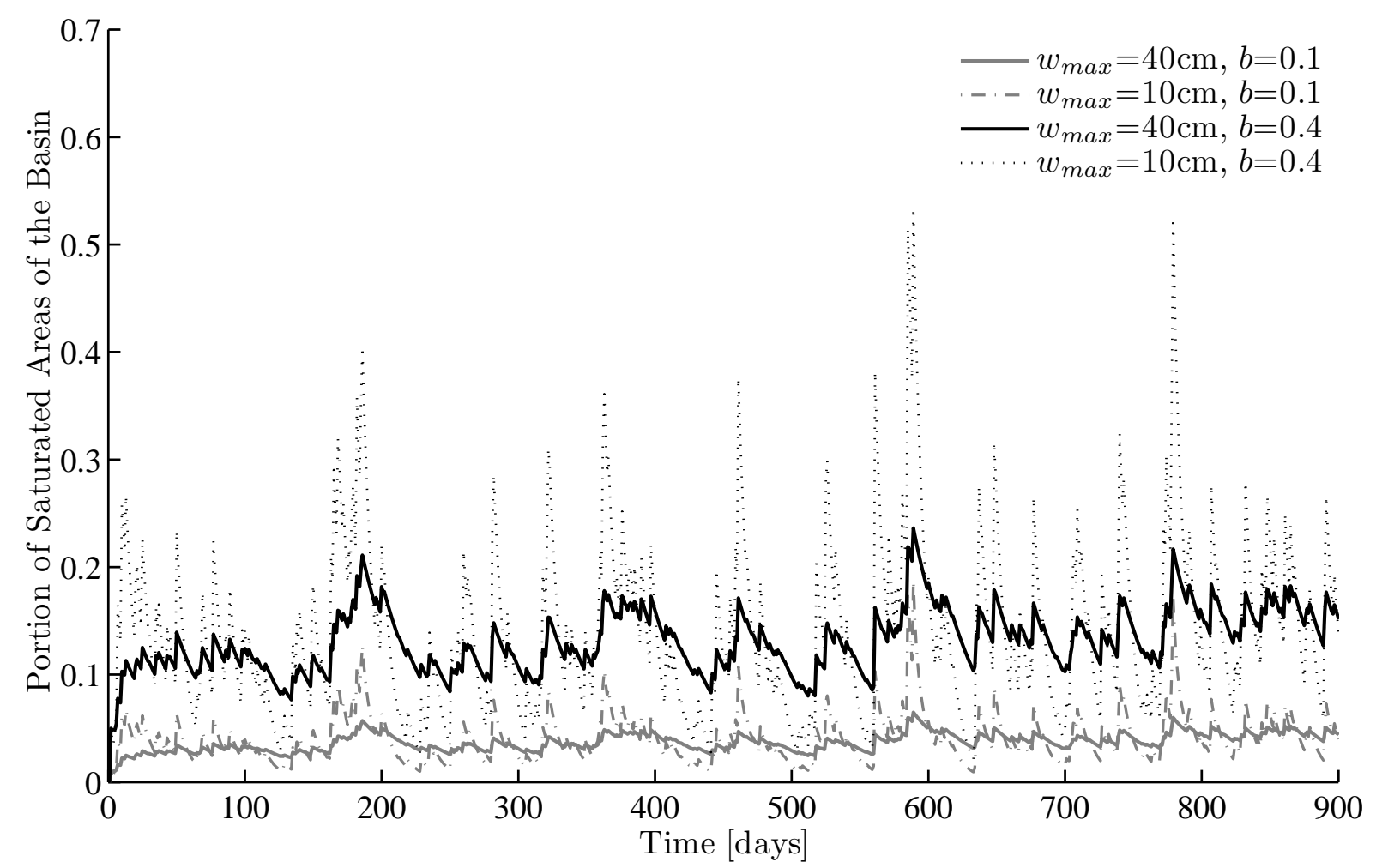

Fig. 4. Temporal dynamics of saturated areas for different values of $w_{\max }$ and $b$ reproduced by a numerical simulation performed at the daily time-scale. The remaining parameters are $\lambda=0.3, \alpha=1.0 \mathrm{~cm}$ and $V=0.7 \mathrm{~cm} /$ day.

3.1 Probability density function of saturated areas of the basin

Under these hypotheses, it is possible to define the probability distribution of saturated areas given the climatic forcing and the geomorphologic characteristics of the basin. In particular, the probability density function of $a$ can be obtained from the probability density function of $R$ as

$$
p_{A}(a)=p_{R}\left(f^{-1}(a)\right) \frac{d f^{-1}(a)}{d a} .
$$

To this end, it is necessary to clarify the relationship between $R$ and $a$ that may be obtained from Eq. (2) where the ratio $f / F$ may be also interpreted as the saturated portion of the basin. It follows

$$
R=f^{-1}(a)=1-(1-a)^{\frac{1}{b}} .
$$

The derivative of $f^{-1}(a)$ is

$\frac{d f^{-1}(a)}{d a}=\frac{1}{b}(1-a)^{\frac{1}{b}-1}$.
Consequently, using the Eq. (19) one obtains the following expression for the probability density function of the relative saturated areas of a basin

$$
\begin{aligned}
& p_{A}(a)=\frac{(1-a)^{\frac{1}{b}-1}}{b \beta} C e^{\gamma\left((1-a)^{\frac{1}{b}}-1\right)-(1-a)^{\frac{1}{b}} k} . \\
& \left(e^{k}-1\right)\left(e^{k-(1-a)^{\frac{1}{b}} k}-1\right)^{\frac{\lambda-e^{-k \lambda}}{k \beta}-1} .
\end{aligned}
$$

3.2 Probability density function of the relative saturation of the basin

The relative saturation of the basin can be easily characterized at this point using the probability distribution of Eq. (14). Then, one can use the relationship between $R$ and $s$,

$$
R=g^{-1}(s)=\left(1-(1-s)^{\frac{1}{1+b}}\right),
$$

to obtain the derived probability distribution of $s$. To this end, the same approach used in the previous paragraph should 
be used where one also need the derivative of the function $g^{-1}(s)$

$\frac{d g^{-1}(s)}{d s}=\frac{(1-s)^{\frac{1}{1+b}-1}}{1+b}$.

The probability density function for the relative saturation of the basin at the steady state can be described by the following expression

$$
\begin{aligned}
& p(s)=\frac{(1-s)^{\frac{-b}{1+b}} C}{(1+b) \beta}\left(e^{k}-1\right)\left(e^{\left.k-k(1-s)^{\frac{1}{1+b}}-1\right)^{\frac{\lambda-e^{-k \lambda}}{k \beta}-1} .}\right. \\
& e^{-k(1-s)^{\frac{1}{1+b}}+\gamma\left((1-s)^{\frac{1}{1+b}}-1\right)} .
\end{aligned}
$$

\section{Results and discussion}

The model proposed here is a minimalist representation of soil moisture dynamics at basin scale. In the following applications the derived PDFs are tested using Monte Carlo simulations in order to understand the reliability of the adopted simplification for the analytical derivation and also to evaluate the ability of the model to reproduce the dynamics of a river basin. Results show that the model provides a realistic description of the basin water balance under a wide range of climatic and physical conditions.

In order to show the dynamics of the relative saturated portion of the basin, a numerical simulation of the described model with no approximation in the soil water loss function was performed over a temporal window of 100 years using different values of $b$ and $w_{\max }$. A realization of the process is given in Fig. 4 considering a limited temporal window of 900 days. Different parameters of the soil water storage capacity distribution may change dramatically the dynamics of the system and this is even more clear in the PDFs of $a$ and $s$ described in the following paragraphs. The simulation has been used for comparison with the theoretical distributions obtaining a very good agreement as one may observe in Fig. 5. The two probability density functions of the relative saturation and the relative portion of the saturated areas of the basin are compared with the PDFs obtained via numerical simulation. This result provides an idea of the errors associated with the use of an approximated function to describe the soil water losses (see Eq. 9).

Figure 6 describes a sequence of probability distributions of the relative saturation of the basin and of the saturated areas assuming different set of parameters for the distribution of water storage capacity with fixed climatic conditions. It may be immediately appreciated how the relative structure of the basin plays a fundamental role in the dynamics of $s$ and $a$. It is interesting to note that on one hand the reduction of the maximum water storage capacity, $w_{\max }$, increases the variability of both relative basin saturation and saturated
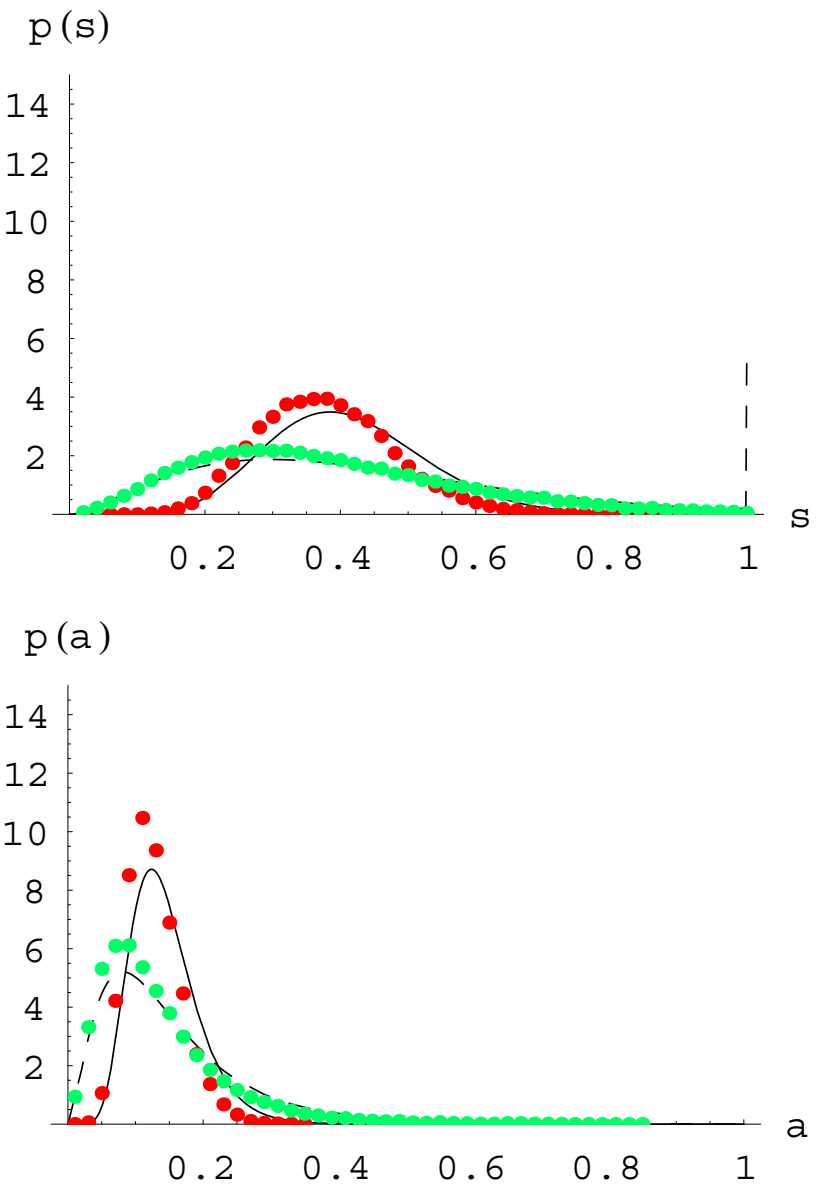

Fig. 5. Comparison between the probability density functions of the relative saturation of the basin (upper graph) and relative saturated areas (bottom) obtained with the theoretical distribution given in Eqs. (21) and (24) and numerical simulation (full circles). The parameters adopted are $w_{\max }=40 \mathrm{~cm}$ (continuous line) and $10 \mathrm{~cm}$ (dashed line), while the remaining are $b=0.4, \lambda=0.3, \alpha=1.0 \mathrm{~cm}$ and $V=0.7 \mathrm{~cm} /$ day.

areas; on the other hand the increase of the heterogeneity in the distribution of $W$, dictated by the parameter $b$, does not provide significant change on $s$. In fact, the increase of the exponent $b$ does not apparently affect the variance of $s$, but at the same time it increases the mean and the variability of the saturated areas.

The role of climatic forcing is described in Fig. 7, where some examples of PDFs, derived from Eqs. (21) and (24), are plotted for different values of the parameters $\alpha$ and $\lambda$. These two parameters control the mean rainfall rate $(\alpha \lambda)$ and their increase with a fixed value of $V$ means a shift towards more humid environments. Such a variation in the climatic conditions reflects on the position and also on the shape of the PDFs of both $s$ and $a$. In the examined cases, changes in the mean rainfall depth, $\alpha$, produce a more 

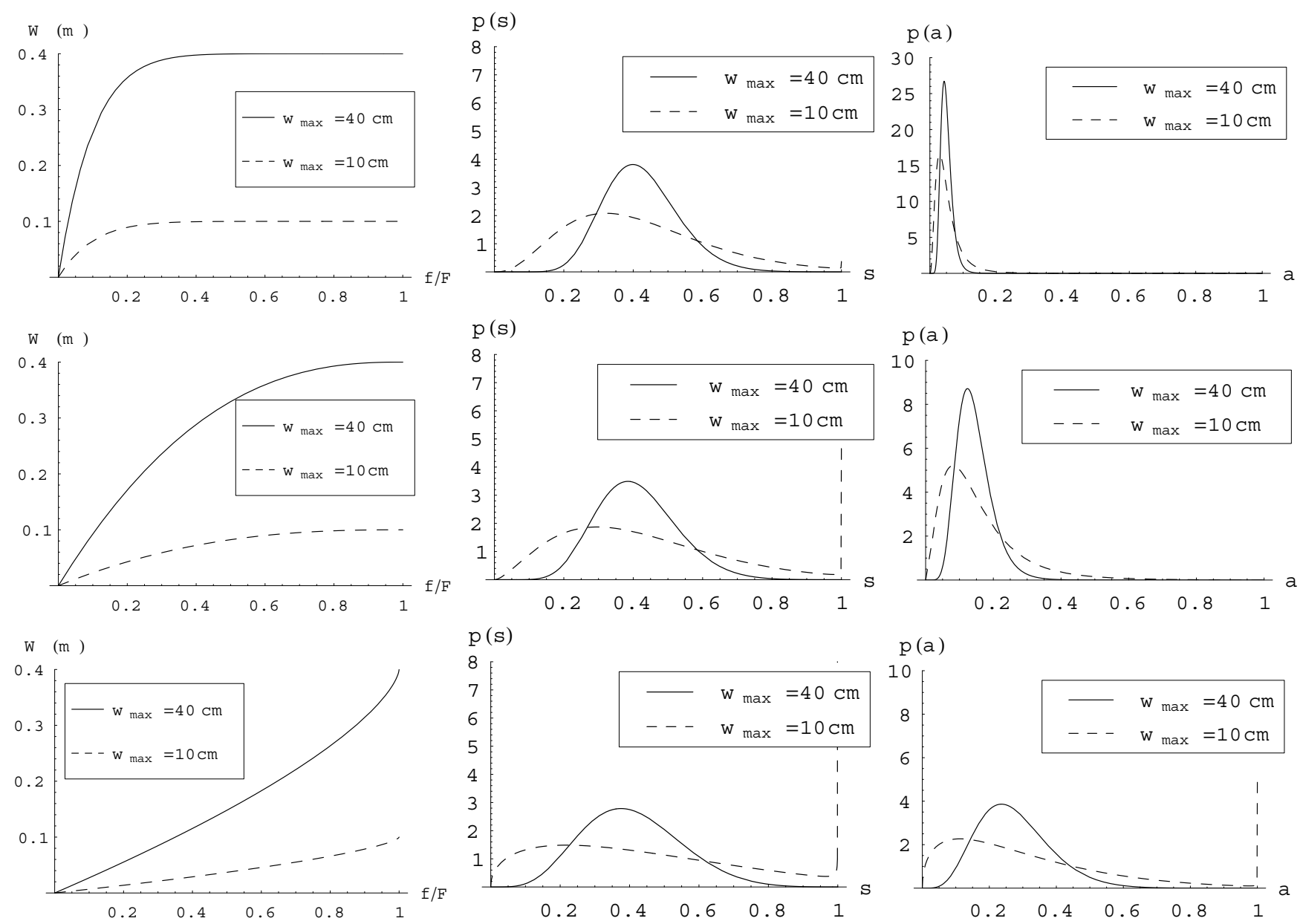

Fig. 6. Probability density functions of the relative saturation (second column) and of the saturated areas (third column) of a river basin assuming $w_{\max }$ equal to $40 \mathrm{~cm}$ and $10 \mathrm{~cm}$, while the parameter $b$ varies between $0.1,0.4$ and 1.5 in the top down order. The remaining parameters are $\lambda=0.3, \alpha=1.0 \mathrm{~cm}$ and $V=0.7 \mathrm{~cm} /$ day. In the first column, the soil water storage capacity distribution is represented for the corresponding set of parameters $w_{\max }$ and $b$ on each row.

marked change in the PDF's shape even under the same mean rainfall rate. In fact, one may compare the dashed PDF in the first panel on the left, obtained assuming the parameters $\alpha=20 \mathrm{~mm}$ and $\lambda=0.1$ event/day producing a mean rainfall rate of $2 \mathrm{~mm} /$ day, with the PDF in the second panel on the left (continuous line) obtained with parameters $\alpha=10 \mathrm{~mm}$ and $\lambda=0.2$ event/day characterized by the same rainfall rate. The two distribution are slightly different and in particular it seems that the increase in the mean rainfall depth of the storms increases the variability of the relative saturation of the basin. This effect is even more marked in the PDFs of relative saturated areas.

The mean and the standard deviation of the saturated areas, $a$, are described in Fig. 8 as a function of the water loss coefficient $V$. Generally, the mean value of the basin saturated areas decrease with the increase of the water loss coefficient. A different behaviour is observed for the standard deviation that reaches a maximum value when the soil water losses coefficient is equal to the mean daily rainfall (here equal to $\alpha \lambda=3 \mathrm{~mm}$ ). One may also observe that the presence of a more heterogeneous soil water storage capacity $(b=0.4)$ induces a higher mean but also a higher variability.

The parameters $w_{\max }$ and $b$ may also affect the partition between runoff and soil water losses that is described in Fig. 9 as a function of the Poisson rate of rainfall $\lambda$. The general signal is an increase of the soil losses with the increase of the incoming rainfall. Of course some differences may be observed for the different basin configuration considered herein. It may be noticed that the increase of the parameter $b$, representing the spatial heterogeneity of the water storage capacity, tends to reduce the expected value of the soil water losses, but certainly in this case the controlling parameter is the maximum value of the water storage capacity $w_{\max }$. 

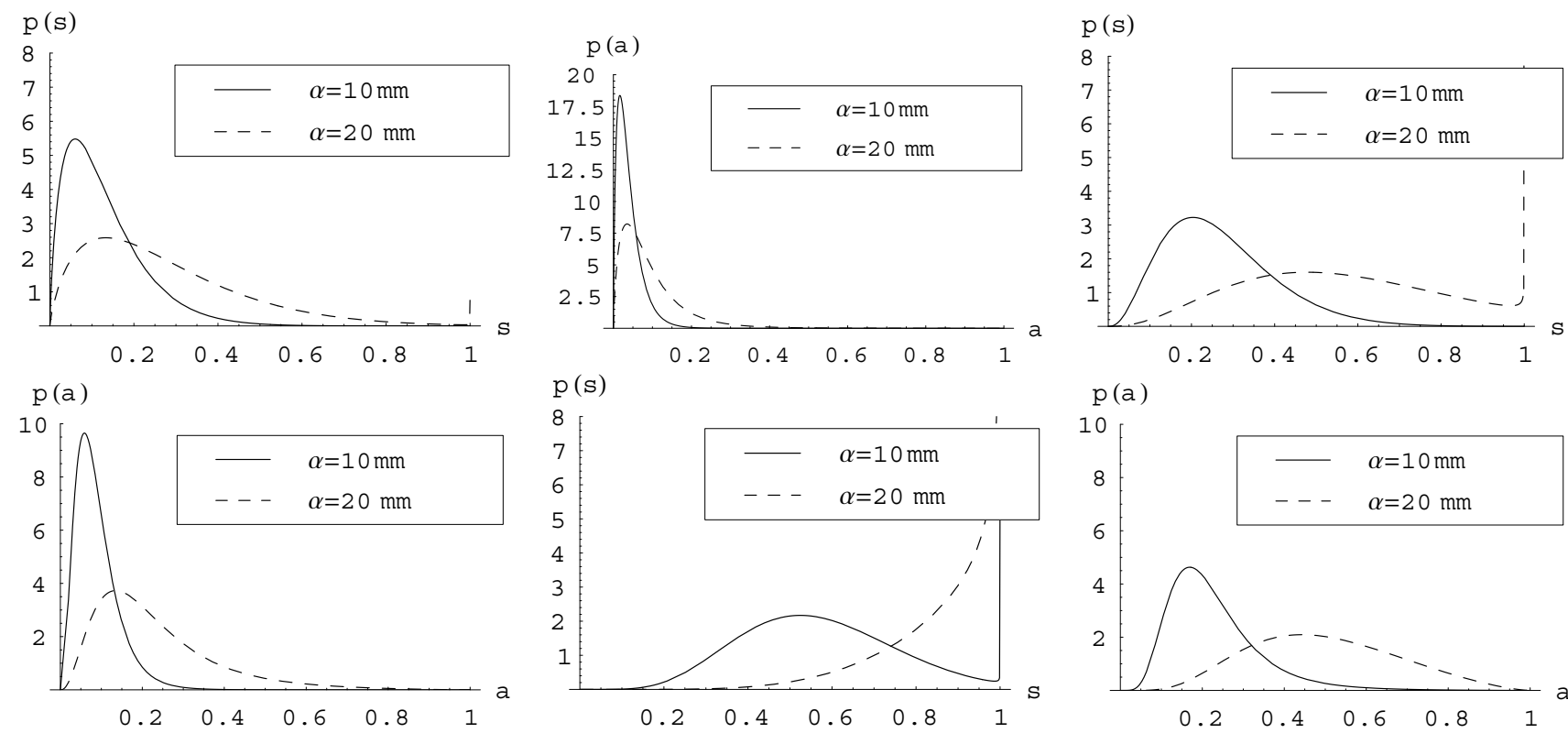

Fig. 7. Probability density functions of the relative saturation (first column) and of the saturated areas (second column) of a river basin assuming $w_{\max }$ equal to $20 \mathrm{~cm}$, the parameter $b=0.4$ and two different values for $\alpha(10 \mathrm{~mm}$ and $20 \mathrm{~mm})$, while the parameter $\lambda$ varies between $0.1,0.2$ and 0.4 in the top-down order.
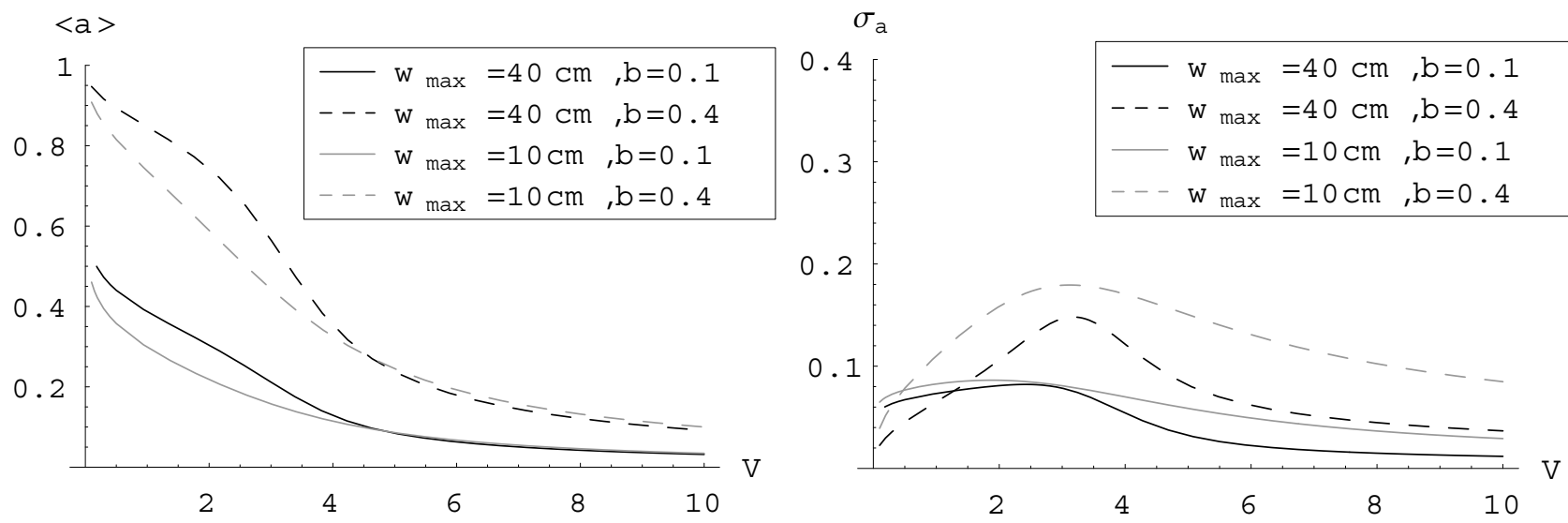

Fig. 8. Expected value and standard deviation of the saturated areas of the basin as a function of the soil water loss coefficient, $V$, for different values of $w_{\max }$ and $b$. Remaining parameters are the same of Fig. 6.

\subsection{Comparison of the theoretical model with a continuous numerical simulations}

The model has been tested using the data obtained from a continuous hydrological simulation performed using a semi-distributed hydrological model (DREAM - Manfreda et al., 2005) in cascade with a rainfall generator (IRP - Veneziano et al., 2002). Montecarlo simulations were performed over 800 years using synthetic rainfall spatially uniform over the basin. Results were used by Fiorentino et al. (2007) to derive the probability distribution of the runoff contributing areas during rainfall events for the Agri and the Bradano river basins (Southern Italy). These areas also represent the saturated portion of the basin since the DREAM model adopts a runoff generation based on saturation excess and the rainfall was assumed to be uniform in the modelling application. For these reasons, the results of the work by Fiorentino et al. (2007) represent an ideal dataset to test the 
proposed theoretically derived PDFs.

Among the two basins investigated by Fiorentino et al. (2007), the Agri represents a perfect study case belonging to a humid area suitable to be interpreted through the proposed mathematical model. This basin has been deeply investigated in previous studies and its detailed description is available in Fiorentino et al. (2006, 2007). Consequently, the modelling results obtained for this basin are used here to test the reliability and applicability of the probability distribution of saturated areas derived in the present paper.

Parameters of the theoretical distribution have been computed exploiting as much as possible the available information on the Agri River basin. In particular, rainfall parameters have been estimated from rainfall records during the wet season, the parameter $V$ is estimated from the equation proposed by Pan et al. (2003),

$V=\max \left(1,6.08+0.40 K_{s}-0.51 L A I\right)[\mathrm{mm} /$ day $]$,

where $K_{s}=6.06 \mathrm{~cm} / \mathrm{h}$ (mean value of the permeability over the basin) and $\mathrm{LAI}=1.28$ (mean value over the basin during the wet season). The parameter $b$ was fitted using the method proposed by Chen et al. (2007) exploiting the topographic index computed from a digital elevation model at $240 \mathrm{~m}$ of resolution obtaining an estimate of $b=0.39$.

The comparison between the PDFs of saturated areas obtained with the two procedures is depicted in Fig. 10 where the theoretical distributions have been plotted using two different values for the parameter $w_{\max }$ derived from Eq. (3) using the two extremes that the total water storage capacity, $W M$, can assume according to Zhao (1984) and Zhao and Wang (1988). Both the theoretical PDFs have a good agreement with the results obtained from the simulation performed with the DREAM model. Of course, the semi-empirical parameter $w_{\max }$ should be estimated from runoff data in order to get a more accurate estimate of $p(a)$, but this preliminary results shows a low variability of this distribution respect to the range of variability assumed by this last parameter.

\section{Conclusions}

In the present paper, a new approach is introduced to describe analytically the relative soil saturation of a river basin and the dynamics of its saturated areas. The method provides a simplified description of river basin characteristics, but includes the effect of spatial variability of water storage capacity adopting the same schematization used by Zhao et al. (1980) for the Xinanjiang model.

In summary, this approach allowed to:

- Derive analytically the probability density function of the saturated portion of a basin also called runoff source areas that represent a significant variable in the dynamics of a river basin (e.g. Fiorentino et al., 2007). Furthermore, the model introduced may be easily adopted

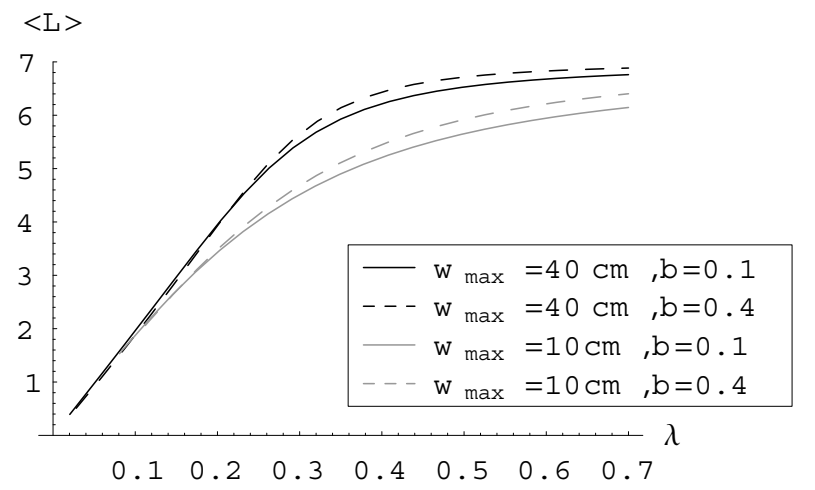

Fig. 9. Expected value of the soil water losses $<L>$ as a function of the parameter $\lambda$ assuming different values for $w_{\max }$ and $b$. The remaining parameters are $\lambda=0.3, \alpha=2.0 \mathrm{~cm}$ and $V=0.7 \mathrm{~cm} /$ day.

to derive the probability density function of runoff production as it will be described in a subsequent paper by Manfreda $(2008)^{1}$.

- Derive the probability density function of the relative saturation of a river basin characterized by a given climatic forcing and distribution of the soil water storage capacity.

- Identify the role of climatic and physical features of the basin on its soil water dynamics in humid environments through the use of physically meaningful parameters $(\alpha$, $\lambda$ and $V$ ) and semi-empirical parameters $\left(b\right.$ and $\left.w_{\max }\right)$. In this context, an interesting results was observed in the variability of saturated areas that apparently reached its maximum when the soil water loss coefficient gets close to the mean rainfall rate.

- Understand the role played by the distribution of the soil water storage capacity on soil water of the basin. In particular, results outlined a strong control of the spatial heterogeneity on the shape of the probability distribution of saturated areas, while the relative saturation of the basin seems more controlled by the maximum water storage capacity.

- Define a theoretical framework useful also for the developers and numerous users of the Xinanjiang model and similar conceptual models.

The model has been tested with the results of a continuous numerical simulation performed with a distributed model obtaining a good agreement between the two outcomes. The exercise reported here was particularly useful to design a strategy for the parameter estimation of the model that turn out to be straightforward.

\footnotetext{
${ }^{1}$ Manfreda, S.: Runoff Production Dynamics within a Humid River Basin, Nat. Hazard Earth Syst. Sci., submitted, 2008.
} 


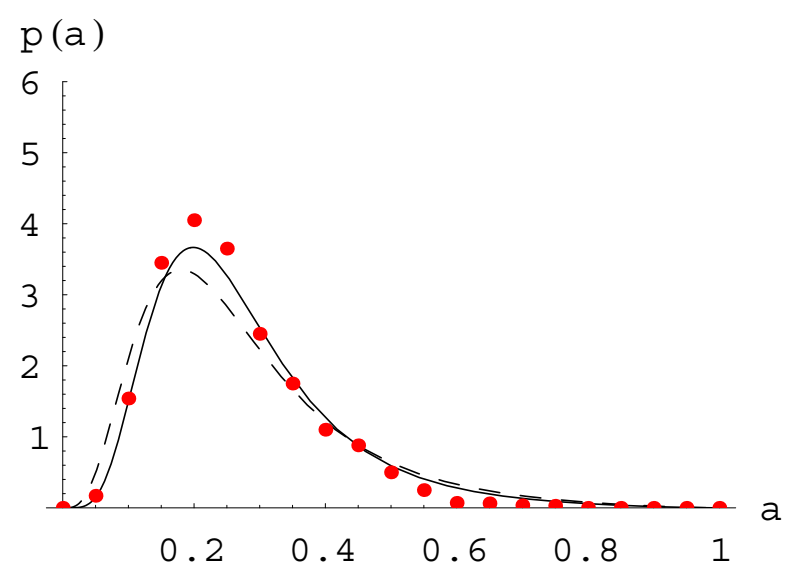

Fig. 10. Comparison between the probability distribution of the saturated areas of the Agri River basin obtained from numerical simulation with a semi-distributed model (full circles) and the theoretical density functions derived in the present work where the parameters are: $\lambda=0.29, \alpha=1.35 \mathrm{~cm}, V=0.606 \mathrm{~cm} /$ day, $b=0.39$ and finally $w_{\max }$ is assumed equal to $16.6 \mathrm{~cm}$ (dashed line) and $22.2 \mathrm{~cm}$ (continuous line).

The model has not been applied to a real case yet, but a specific experiment has been designed in order to derive the statistics of the averaged soil moisture over a basin hillslope in order to compare the derived PDFs to a real case. Moreover, the proposed scheme can be used to derive the probability density function of the runoff production at basin scale taking into to account two relevant phenomena like the non-linearity in the rainfall-runoff generation mechanisms and the saturation effect of the basin (Manfreda, 2008 ${ }^{1}$ ).

\section{Appendix A}

\section{Notation}

$a$ fraction of saturated areas [dimensionless].

$C$ constant of integration [dimensionless].

$F_{1}[., ., .,$.$] hypergeometric function.$

$f / F$ saturated portion of the basin [dimensionless].

$\Gamma[$.$] complete gamma function.$

$I$ infiltration $[\mathrm{cm}]$.

$L_{b}(R)$ soil water loss function at the basin scale $\left[\mathrm{cm} \mathrm{d}^{-1}\right]$. $R=\frac{w m_{t}}{w_{\max }}$ relative water level in the basin [dimensionless].

$s$ relative saturation of the basin [dimensionless].

$k$ coefficient of the simplified soil water loss function used to fit Eq. (7) [dimensionless].

$W$ water storage capacity at a point $[\mathrm{cm}]$.

$W_{I}$ wetness index $[\ln (\mathrm{m})]$.

$w_{\max }$ maximum value of the water storage capacity in the basin $[\mathrm{cm}]$.

$w m_{t}$ water level in the parabolic reservoir $[\mathrm{cm}]$.
$W_{t}$ total water content $[\mathrm{cm}]$.

$V$ water loss coefficient $\left[\mathrm{cm} \mathrm{d}^{-1}\right]$.

$\beta=V /\left(w_{\max }\right)$ is the normalized soil water loss coefficient [dimensionless].

$\gamma=w_{\max } / \alpha$ is the normalized mean rainfall depth

[dimensionless].

$\alpha$ mean depth of rainfall events $[\mathrm{cm}]$.

$\lambda$ rainfall rate per unit time $\left[\mathrm{d}^{-1}\right]$.

$\rho(R)$ simplified water loss function.

Acknowledgements. This study was supported by the MIUR (Italian Ministry of Instruction, University and Research) under the grant PRIN CoFin2005 entitled "Climate-soil-vegetation interaction in hydrological extremes". S. Manfreda gratefully acknowledges the support of the CARICAL foundation for his research activities.

Edited by: M. Sivapalan

\section{References}

Abramowitz, M. and Stegun, I. A.: Handbook of Mathematical Functions with Formulas, Graphs, and Mathematical Tables, New York, Dover, 1046 pp., 1964.

Arnold, L.: Stochastic Differential Equations: Theory and Applications, John Wiley \& Sons, New York, 228 pp., 1974.

Beven, K. J. and Kirkby, M. J.: A physically-based variable contributing area model of basin hydrology, Hydrol. Sci. B., 24(1), 43-69, 1979.

Botter, G., Porporato, A., Rodríguez-Iturbe, I., and Rinaldo, A.: Basin-scale soil moisture dynamics and the probabilistic characterization of carrier hydrologic flows: leaching-prone components of the hydrologic responce, Water Resour. Res., 43, W02417, doi:10.1029/2006WR005043, 2007a.

Botter, G., Porporato, A., Daly, E., Rodríguez-Iturbe, I., and Rinaldo, A.: Probabilistic characterization of base flows in river basins: Roles of soil, vegetation, and geomorphology, Water Resour. Res., 43, W06404, doi:10.1029/2006WR005397, 2007b.

Caylor, K. K., Manfreda, S., and Rodríguez-Iturbe, I.: On the coupled geomorphological and ecohydrological organization of river basins, Adv. Water Resour., 28(1), 69-86, 2005.

Chen, X., Chen, Y. D., and Xu, C.-Y.: A distributed monthly hydrological model for integrating spatial variations of basin topography and rainfall, Hydrol. Process., 21(2), 242-252, 2007.

Dunne, T. and Black, R.: An Experimental Investigation of Runoff Production in Permeable Soils, Water Resour. Res., 6(2), 478490, 1970.

Entekhabi, D. and Rodríguez-Iturbe, I.: An analytic framework for the characterization of the space-time variability of soil moisture, Adv. Water Resour., 17, 25-45, 1994.

Fiorentino, M., Gioia, A., Iacobellis, V., and Manfreda, S.: Analysis on flood generation processes by means of a continuous simulation model, Adv. Geosci., 7, 231-236, 2006, http://www.adv-geosci.net/7/231/2006/.

Fiorentino, M., Manfreda, S., and Iacobellis, V.: Peak Runoff Contributing Area as Hydrological Signature of the Probability Distribution of Floods, Adv. Water Resour., 30, 2123-2134, 2007. 
Guo, F., Liu, X. R., and Ren, L. L.: Topography based watershed hydrological model-TOPOMODEL and its application, Advances in Water Science, 11(3), 296-301, 2000 (in Chinese).

Hewlett, J. D. and Hibbert, A. R., Factors affecting the response of small watersheds to precipitation in humid areas, in: Forest Hydrology, edited by: Sopper, W. E. and Lull, H. W., Pergamon Press, 275-290, 1967.

Isham, V., Cox, D. R., Rodríguez-Iturbe, I., Porporato, A., and Manfreda, S.: Mathematical characterization of the space-time variability of soil moisture, Proc. R. Soc. Lon. Ser.-A., 461(2064), 4035-4055, 2005.

Jawson, S. D. and Niemann, J. D.: Spatial patterns from EOF analysis of soil moisture at a large scale and their dependence on soil, land-use, and topographic properties, Adv. Water Resour., 30(3), 366-381, 2007.

Kim, G. and Barros, A. P.: Space-time characterization of soil moisture from passive microwave remotely sensed imagery and ancillary data, Remote Sens. Environ., 81, 393-403, 2002.

Laio, F., Porporato, A., Fernandez-Illescas, C. P., and RodríguezIturbe, I.: Plants in water-controlled ecosystems: Active role in hydrologic processes and response to water stress, IV: Discussion of real cases, Adv. Water Resour., 24, 745-762, 2001.

Manfreda, S., Fiorentino, M., and Iacobellis, V.: DREAM: a distributed model for runoff, evapotranspiration, and antecedent soil moisture simulation, Adv. Geosci., 2, 31-39, 2005, http://www.adv-geosci.net/2/31/2005/.

Manfreda, S., Porporato, A., and Rodríguez-Iturbe, I.: Dinamiche spazio-tempo dell'umidità del suolo: la struttura stocastica ed il campionamento, Giornata di Studio: Metodi Statistici e Matematici per l'Analisi delle Serie Idrologiche, edit by: Piccolo, D. and Ubertini, L., Viterbo, 25-38, 2006 (in Italian).

Manfreda, S. and Rodríguez-Iturbe, I.: On the Spatial and Temporal Sampling of Soil Moisture Fields, Water Resour. Res., 42, W05409, doi:10.1029/2005WR004548, 2006.

Montaldo, N., Rondena, R., and Albertson, J. D.: Parsimonious modeling of vegetation dynamics for ecohydrological studies of water-limited ecosystems, Water Resour. Res., 41, W10416, doi:10.1029/2005WR004094, 2005.

Moore, R. J. and Clarke, R. T.: A distribution function approach to rainfall runoff modelling, Water Resour. Res., 17, 1367-1382, 1981.

Moore, R. J.: The probability-distributed principle and runoff production at point and basin scales, Hydrol. Sci., 30, 273-297, 1985.

Moore, R. J.: Real-time flood forecasting system: perspectives and prospects, in: Flood and landslides: Integrated risk assessment, edited by: Casal, R. and Margottini, C., Springer, 147-189, 1999.

Pan, F., Peters-Lidard, C. D., and Sale, M. J.: An analytical method for predicting surface soil moisture from rainfall observations, Water Resour. Res., 39(11), 1314, doi:10.1029/2003WR002142, 2003.

Porporato, A., Daly, E., and Rodríguez-Iturbe, I.: Soil water balance and ecosystem response to climate change, Am. Nat., 164(5), 625-633, 2004.

Porporato, A., Laio, F., Ridolfi, L., and Rodríguez-Iturbe, I.: Plants in water controlled ecosystems: Active role in hydrological processes and response to water stress, III: Vegetation water stress, Adv. Water Resour., 24, 725-744, 2001.

Prudnikov, A. P., Brychkov, Y. A., and Marichev, O. I.: Integrals and Series, Volume 1, Elementary Functions, Gordon and Breach Science Publishers, New York, 798 pp., 1986.

Rigby, J. R. and Porporato, A.: Simplified stochastic soil-moisture models: a look at infiltration, Hydrol. Earth Syst. Sci., 10, 861871, 2006,

http://www.hydrol-earth-syst-sci.net/10/861/2006/.

Rodríguez-Iturbe, I. and Porporato, A.: Ecohydrology of Watercontrolled Ecosystems: Soil Moisture and Plant Dynamics, Cambridge University Press, Cambridge, UK, 460 pp., 2004.

Rodríguez-Iturbe, I., Porporato, A., Ridolfi, L., Isham, V., and Cox, D. R.: Probabilistic modelling of water balance at a point: The role of climate, soil and vegetation, Proc. R. Soc. Lon. Ser.-A., 455, 3789-3805, 1999.

Rodríguez-Iturbe, I., Isham, V., Cox, D. R., Manfreda, S., and Porporato, A.: Space-time modeling of soil moisture: Stochastic rainfall forcing with heterogeneous vegetation, Water Resour. Res., 42, W06D05, doi:10.1029/2005WR004497, 2006.

Scanlon, T. M., Caylor, K. K., Manfreda, S., Levin, S. A., and Rodríguez-Iturbe, I.: Dynamic Response of Grass Cover to Rainfall Varyiability: Implications the Function and Persistence of Savanna Ecosystems, Adv. Water Resour., 28(3), 291-302, 2005.

Sivapalan, M. and Woods, R. A.: Evaluation of the effects of general circulation models' subgrid variability and patchiness of rainfall and soil moisture on land surface water balance fluxes, Hydrol. Process., 9, 697-717, 1995.

Sivapalan, M., Woods, R. A., and Kalma, Y. D.: Variable bucket representation of topmodel and investigation of the effects of rainfall heterogeneity, Hydrol. Process., 11, 1307-1330, 1997.

Todini, E.: The ARNO rainfall-runoff model, J. Hydrol., 175, 339$382,1996$.

Western, A. W., Grayson, R. B., and Blöschl, G.: Scaling of soil moisture: A hydrologic perspective, Annu. Rev. Earth Pl. Sc., 30, 149-180, 2002.

Wilson, D. J., Western, A. W., and Grayson, R. B.: Identifying and quantifying sources of variability in temporal and spatial soil moisture observations, Water Resour. Res., 40, W02507, doi:10.1029/2003WR002306, 2004.

Wood, E. F., Lettenmaier, D. P., and Zartarian, V. G.: A land-surface hydrology parametrization with subgrid varibility for general circulation models, J. Geophys. Res., 97, 2717-2728, 1992.

van Wijk, M. T. and Rodríguez-Iturbe, I.:Tree-grass competition in space and time: Insights from a simple cellular automata model based on ecohydrological dynamics, Water Resour. Res., 38(9), 1179, doi:10.1029/2001WR000768, 2002.

Veneziano, D., Furcolo, P., and Iacobellis, V.: Multifractality of iterated pulse processes with pulse amplitudes generated by a random cascade, Fractals, 10(2), 209-22, 2002.

Zhao, R.-J. and Wang P. L.: Parameter determination of Xinanjiang model, Hydrology, 6, 2-9, 1988 (in Chinese).

Zhao, R.-J., Zhang, Y. L., and Fang, L. R.: The Xinanjiang model, Hydrological Forecasting Proceedings Oxford Symposium, IAHS Publication No. 129, 351-356, 1980.

Zhao, R.-J.: The Xinanjiang model applied in China, J. Hydrol., 135, 371-381, 1992.

Zhao, R.-J.: Water Hydrological Modeling-Xinanjiang Model and Shanbei Model, China Water Resources and Hydropower Publishing House, Beijing, 125-129, 1984 (in Chinese). 